\title{
Pharmacokinetics of Hydrochlorothiazide, Losartan and E3174 after Oral Doses of Losartan and Losartan/Hydrochlorothiazide in Healthy Chinese Male Volunteers"
}

\author{
Sha Liu ${ }^{1,2}$, Fan-Long Bu ${ }^{1}$, Chun-Min Wei ${ }^{1}$, Gui-Yan Yuan ${ }^{1}$, Ben-Jie Wang ${ }^{1}$, Rui-Chen Guo ${ }^{1 \#}$ \\ ${ }^{1}$ The Institute of Clinical Pharmacology, Qilu Hospital of Shandong University, Jinan, China; ${ }^{2}$ Medical Faculty of Shandong Univer- \\ sity, Jinan, China. \\ Email: "grc7636@126.com
}

Received October1 $14^{\text {th }}, 2011$; revised November $20^{\text {th }}, 2011$; accepted December $10^{\text {th }}, 2011$

\begin{abstract}
Aims: A simple and highly sensitive LC-MS method was used to determine the concentrations of losartan, its major active metabolite E3174 and hydrochlorothiazide in human plasma and pharmacokinetic characteristics and metabolism phenotype observation after administration of losartan tablets and losartan/hydrochlorothiazide combination tablets. Methods: An open, randomized cross-over single-dose study was designed in forty healthy male volunteers, A single-dose of $50 \mathrm{mg}$ losartan tablets or $50 \mathrm{mg}$ losartan/12.5 mg hydrochlorothiazide combination tablets was orally given and blood samples were collected at scheduled time. A LC-MS method was established and evaluated for determining the plasma concentrations of losartan, E3174 and hydrochlorothiazide. The pharmacokinetic parameters and characteristic of metabolism phenotypes were calculated and compared via this test. Results: The elimination rate of E3174 $\left(\mathrm{t}_{1 / 2}\right)$ was significantly shorter $(\mathrm{P}<0.05)$ and the $\mathrm{AUC}_{0-t}$ was lower $(\mathrm{P}<0.05)$ for administration of combination tablets. The rest of pharmacokinetic parameters of losartan and E3174 have no significant differences. Among all subjects, three phenotypes were observed: the poor, the intermediate and the extensive metabolism. Conclusion: It is indicated that the test and reference tablets were bioequivalent, and that hydrochlorothiazide could significantly alter the $\mathrm{t}_{1 / 2}, \mathrm{AUC}_{0-\mathrm{t}}$ and $\mathrm{AUC}_{0 \text {-inf }}$ of E3174.
\end{abstract}

Keywords: Losartan; E3174; Hydrochlorothiazide; Pharmacokinetic; Phenotype; LC-MS

\section{Introduction}

Losartan, 2-n-butyl-4-cholo-5-hydroxymethyl-1-[(2\%- $(1 \mathrm{H}-$ tetrazol-5-yl) biphenyl-4-yl)methyl] imidazole (Figure 1), an active and highly specific non-peptide angiotensin II receptor blocker, is applied for treating hypertension in clinic $[1,2]$. After oral administration, losartan undergoes substantial first-pass metabolism by CYP2C9, and then approximately $14 \%$ of it is converted to the pharmacologically active metabolite E3174 (Figure 1). The halflife $\left(t_{1 / 2}\right)$ of E3174 is 10 to 40 times longer than that of losartan. The varied metablism could be induced by interaction of the enzyme activity and different losartan plasma concentration profiles due to different CYP2C9 genotypes [3-7].

Hydrochlorothiazide, a diuretic of the class of ben-

"Project supported by the Major National Science and Technology Project (2012ZX09303-016-003)

"Corresponding author. zothiadiazines, plays a role in the renin angiotensin aldosterone (RAA) system by decreasing active sodium re-absorption and reducing peripheral vascular resistance. It is widely used in pharmaceutical formulations to make single or that combined with other antihypertensive drugs, for treating edema hypertension, diabetes insipidus, hypoparathyroidism, etc. The combination of losartan and hydrochlorothiazide is successfully used in the treatment of hypertension [8].

Mostly, it is believed that the reason of combined application of losartan and hydrochlorothiazide is the interactions between diuretic and angiotensin II receptor blocker. However, it remains unknown about whether the pharmacokinetic parameters of losartan are changed between monotreatment and combined tablets or not. It also remains unclear that hydrochlorothiazide plays a role in the metabolism of losartan and E3174.

Two way crossover blind and randomized studies was applied on healthy Chinese male volunteers following 
<smiles>OCc1c(Cl)nc(Br)n1Cc1ccc(-c2ccccc2-c2nnnnn2)cc1</smiles>

Losartan<smiles></smiles>

EXP 3174

Figure 1. Chemical structures of losartan and E3174.

single-dose losartan tablets or losartan/hydrochlorothiazide combination tablets for pharmacokinetic studies of losartan and its active metabolite E3174 separately [8-13]. The study was approved by the State Food and Drug Administration of China (SFDA, no. 2010L02663). We aim to evaluate the bioequivalence of losartan tablets or losartan/hydrochlorothiazide tablets, compare their pharmacokinetic parameters and examine the metabolism phenotypes in healthy Chinese male volunteers.

\section{Experimental}

\subsection{Materials}

Losartan tablet (50 mg per tablet) was test, and provided by Shandong Lukang Dayu Pharmaceutical Co.LTD. (China). Losartan/ hydrochlorothiazide $(50 \mathrm{mg} / 12.5 \mathrm{mg}$ per tablet) was reference, and provided by Hangzhou MSD Pharmaceutical Co., Ltd. Losartan (batch 100597200501), hydrochlorothiazide (batch 100309-200702), gliclazide (Internal Standard, I.S., batch 100269-9701) and paracetamol (I.S., batch 100018-200408) were obtained from National Institute for the Control of Phar- maceutical and Biological Products. Methanol, acetonitrile and ethyl acetate, HPLC grade, were purchased from J.T.BAKER. Methanoic acid (analytical grade) was obtained from Sinopharm Chemical Reagent Co., Ltd. Blank human plasma was provided by Shandong blood center (P. R.) and was tested for interference before use.

\subsection{Instrument}

LC-MS analyses were performed on an Agilent 1100 Series LC/MSD equipped with an Agilent 1100 Series G1313A autosampler (Agilent Technologies, USA). Stratos type high-speed centrifuge (Kendro, USA), AL104 type electronic balance (Mettler-Toledo Instruments Shanghai Co., Ltd.) and XW-80A type high speed agitator (Shanghai Analysis Instrument Factory) were used. All data were acquired and analyzed with Agilent LC/MSD chemstation analyst data processing software.

\subsection{Analysis Conditions}

The chromatographic conditions were: an Inertsil ${ }^{\circledR}$ ODS-3 column $(150 \mathrm{~mm} \times 4.6 \mathrm{~mm}, 5 \mu \mathrm{m})$ thermostated at $25^{\circ} \mathrm{C}$. The mobile phase of losartan consisted of $0.1 \%$ formic acid-methanol (27:73, V/V, pH2.7), and that of E3174 was $0.1 \%$ formic acid-methanol $(30: 70, \mathrm{~V} / \mathrm{V}, \mathrm{pH} 2.7)$, at a flow rate of $0.8 \mathrm{~mL} \cdot \mathrm{min}^{-1}$. The chromatographic conditions of hydrochlorothiazide were: a Venusil MP C18 column $(250 \mathrm{~mm} \times 4.6 \mathrm{~mm}, 5 \mu \mathrm{m})$ eluted with a mobile phase of water-methanol $(60: 40, \mathrm{~V} / \mathrm{V})$ at $0.6 \mathrm{~mL} \cdot \mathrm{min}^{-1}$.

\subsection{Sample Preparation}

\subsubsection{Losartan}

Plasma $(0.2 \mathrm{~mL})$ spiked with gliclazide (I.S., $20 \mu \mathrm{L})$ was added $600 \mu \mathrm{L}$ of acetonitrile, vortexed for $2 \mathrm{~min}$, and centrifuged at $10800 \mathrm{r} \cdot \mathrm{min}^{-1}$ for $5 \mathrm{~min}$. The supernatant was transferred and $5 \mu \mathrm{L}$ of it was injected for analysis.

Mass spectrometric analysis was performed in the positive ion SIM mode by $\mathrm{m} / \mathrm{z} 423.2$ for losartan and $\mathrm{m} / \mathrm{z}$ 324.2 for gliclazide (I.S.), with spray gas pressure of 50 psi, protective air of nitrogen gas at $11 \mathrm{~L} \cdot \mathrm{min}^{-1}$, capillary voltage of $4000 \mathrm{~V}$, and fragment electric voltage of 100 $\mathrm{V}$ for both.

\subsubsection{E3174}

For E3174 analysis, $20 \mu \mathrm{L}$ of the gliclazide (I.S.) was mixed with $0.5 \mathrm{~mL}$ plasma sample, then $100 \mu \mathrm{L}$ formic acid $(5 \%)$ and $4 \mathrm{~mL}$ ethyl acetate were added, vortexmixed for $2 \mathrm{~min}$, and centrifuged at $5000 \mathrm{rpm}$ for $5 \mathrm{~min}$. The organic phase was transferred to a clean tube and evaporated to dryness under gentle stream of nitrogen gas at $40^{\circ} \mathrm{C}$. The residue was reconstituted with $100 \mu \mathrm{L}$ of mobile phase, and $10 \mu \mathrm{L}$ was injected for LC-MS 
analysis.

Mass spectrometric analysis for E3174 was almost the same as that for losartan, except m/z of E3174 was 437.2.

\subsubsection{Hydrochlorothiazide}

Plasma samples with paracetamol (I.S., $50 \mu \mathrm{L}$ ) were processed by liquid-liquid extraction using ethyl acetate $(4 \mathrm{~mL})$. After vortex-mixed, centrifuged and evaporated to dryness, the residue was reconstituted with $100 \mu \mathrm{L}$ of mobile phase, and then $10 \mu \mathrm{L}$ was injected for LC-MS analysis.

The negtive ion SIM mode was performed in mass spectrometric analysis with $\mathrm{m} / \mathrm{z} 295.7$ for hydrochlorothiazide and $\mathrm{m} / \mathrm{z} 149.9$ for paracetamol. It was same as the above described (2.4.1) for the spray gas pressure, flow rate of protective air of nitrogen gas and capillary voltage. The fragment electric voltages were $120 \mathrm{~V}$ for hydrochlorothiazide and $90 \mathrm{~V}$ for paracetamol.

\subsection{Sample Collections}

Forty healthy adult males with age of 18 - 30 years and BMI of 18 to $25 \mathrm{~kg} / \mathrm{m}^{2}$ were used as subjects involved in the trial, and randomly divided into two groups. Volunteers were chosen according to the medical histories, physical examinations and laboratory tests. Subjects did not take any medication (including Chinese herbal medicine) 4 weeks before or during the study period. Subjects were informed of aims and methods of the study, pharmacological properties of test and reference drugs, adverse reactions and risks by a clinical investigator before submitting written informed consents. The study protocol was approved by the Ethics Committee of the Qilu Hospital of Shandong University in accordance with the Declaration of Helsinki.

After overnight fasting $(10 \mathrm{~h})$, each subject received $50 \mathrm{mg}$ losartan tablets or $50 \mathrm{mg}$ losartan/12.5 mg hydrochlorothiazide combination tablets with $200 \mathrm{ml}$ of water. Food was allowed until $4 \mathrm{~h}$ after administration. Water intake was allowed after $2 \mathrm{~h}$ and low fat standard meals were provided at $4 \mathrm{~h}$ and $10 \mathrm{~h}$ after drug administration. In order to minimize the food effects on the results, subjects received the same standardized foods supplied by nutrition cafeteria of Qilu Hospital of Shandong University.

For losartan group, blood samples were collected at 0 (pre-dose), 0.33, 0.67, 1, 1.33, 1.67, 2.0, 3.0, 4.0, 6.0, 8.0, 10.0, 12.0, 24.0 and $36 \mathrm{~h}$ after administration of losartan tablets. For hydrochlorothiazide combination group, blood samples were collected prior $(0 \mathrm{~h})$ to dosing at 0.33 , $0.67,1,1.33,1.67,2.0,2.5,3.0,4.0,6.0,8.0,10.0,12.0$, $24.0,36.0$ and $48 \mathrm{~h}$ post-dose after administration of 50 $\mathrm{mg}$ losartan/12.5 mg hydrochlorothiazide combination tablets. Both studies had 7 days of washing period. Samples were centrifuged immediately at $3000 \mathrm{rpm}$ for 10 min, and plasma was equally transported into two EP tubes, labeled and stored at $-20^{\circ} \mathrm{C}$ for further analysis.

\subsection{Analysis Method Validation}

The specificity was evaluated by comparing typical chromatograms of losartan (E3174, or hydrochlorothiazide) and I.S. (gliclazide, or paracetamol), blank plasma, blank plasma spiked with losartan (E3174,or hydrochlorothiazide) and I.S. gliclazide (or paracetamol), and plasma spiked with I.S. from subject No. $9,4 \mathrm{~h}$ after administration of losartan/hydrochlorothiazide combination tablets. The extraction recoveries of losartan (E3174, or hydrochlorothiazide) and the I.S. (gliclazide, or paracetamol) were determined by comparing the peak areas of extracted plasma spiked with compound and its I.S. at three quality control (QC) levels with those of post-extraction blank plasma spiked with corresponding concentration of losartan (E3174, or hydrochlorothiazide) and I.S. (gliclazide, or paracetamol). QC samples were 10, $100,750 \mathrm{ng} \cdot \mathrm{mL}^{-1}$ for losartan, $10,100,700 \mathrm{ng} \cdot \mathrm{mL}^{-1}$ for E3174 and 1, 20, $140 \mathrm{ng} \cdot \mathrm{mL}^{-1}$ for hydrochlorothiazide. QC were analyzed before or after freezing at $-20^{\circ} \mathrm{C}$ for $1,7,14$ and longer days (21 days for losartan, 31 days for E3174 and 62 days for hydrochlorothiazide), or all after one or two freeze-thaw circles to evaluate its stability. The Lower Limit of Quantization (LLOQ) was evaluated by analyzing five replicates of spiked plasma samples at $5 \mathrm{ng} \cdot \mathrm{mL}^{-1}$ for both losartan and E3174, or at $1 \mathrm{ng} \cdot \mathrm{mL}^{-1}$ for hydrochlorothiazide. The relative standard deviation (RSD) of QC samples was supposed to be within $15 \%$, while $20 \%$ for RSD of LLOQ. The linearity was observed ranged of $5 \sim 800 \mathrm{ng} \cdot \mathrm{mL}^{-1}, 5 \sim 750 \mathrm{ng} \cdot \mathrm{mL}^{-1}$ and $1 \sim 150 \mathrm{ng} \cdot \mathrm{mL}^{-1}$ for losartan, E3174 and hydrochlorothiazide.

\subsection{Data Processing}

The pharmacokinetic parameters were determined by using non-compartmental model method. The $\mathrm{t}_{1 / 2}, \mathrm{AUC}_{0-\mathrm{t}}$ and $\mathrm{AUC}_{0-\text { inf }}$ were calculated using Drug and Statistical Software-Version 2.0 (DAS 2.0, P.R. of China). Statistical analysis was performed using $\mathrm{T}$-test and F-test and the significance level was set at $P<0.05$. The formula of $\mathrm{AUC}_{\mathrm{E} 3174} / \mathrm{AUC}_{\text {losartan (0-t) }}$ was calculated for investigation of metabolism phenotypes after single oral administration of $50 \mathrm{mg}$ losartan tablets.

\section{Results}

\subsection{Method Validation}

Protonated pseudomolecular ions at ion SIM mode was 
$\mathrm{m} / \mathrm{z} 423.2$ for losartan, m/z 437.2 for E3174, m/z 295.7 for hydrochlorothiazide, $\mathrm{m} / \mathrm{z} 324.2$ for gliclazide (I.S.), and $\mathrm{m} / \mathrm{z} 149.9$ for paracetamol (I.S.), respectively. Figure 2 shows the full-scan mass spectra of all the compounds. Losartan, E3174 and hydrochlorothiazide in

*MSD1 SPC, time=5.640:5.910 of LSTJ2 LLSTJ0000.D API-ES, Pos, Scan, Frag: 100

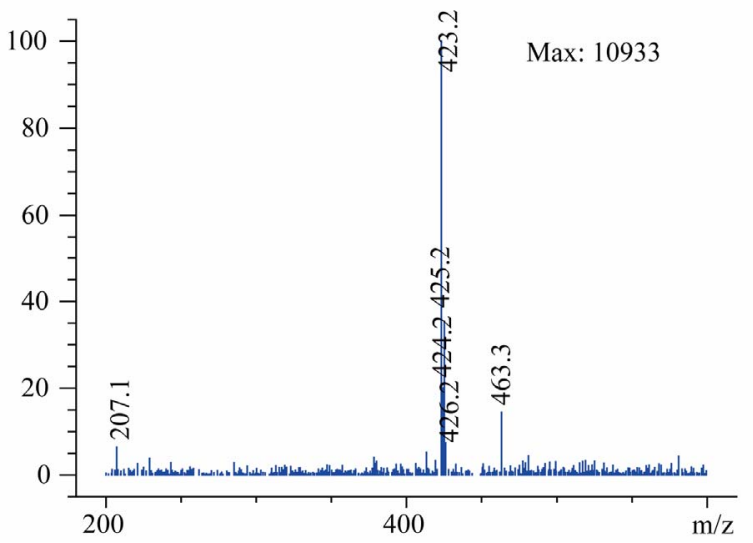

(a)

*MSD1 SPC, time=6.791:7.202 of QLSQ\QLSQ0299.D API-ES, Neg, Scan, Frag: 120

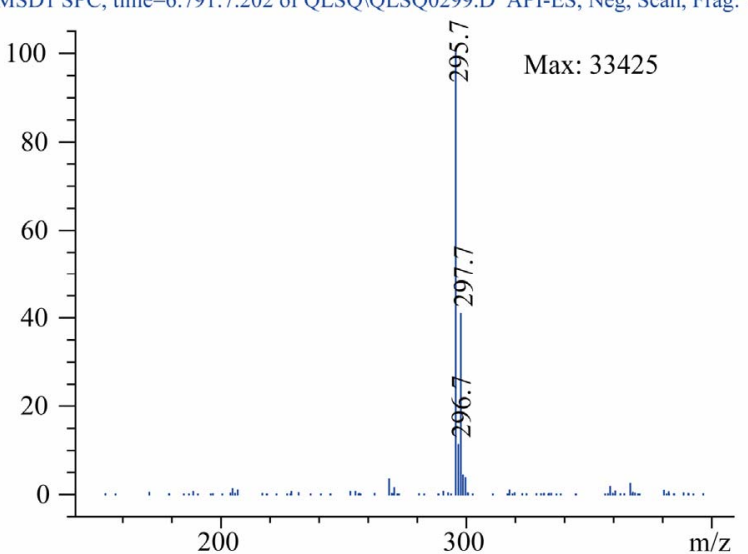

(c) plasma were eluted with retention times of 5.821, 9.902 and $6.949 \mathrm{~min}$, respectively (Figure 3). Gliclazide used as I.S. for losartan and E3174, and paracetamol for hydrochlorothiazide in plasma were eluted with retention times of 5.366, 6.377 and $7.880 \mathrm{~min}$ (Figure 3).

*MSD1 SPC, time=9.437:10.184 of LSTJ2\LSTJ0690.D API-ES, Pos, Scan, Frag: 100

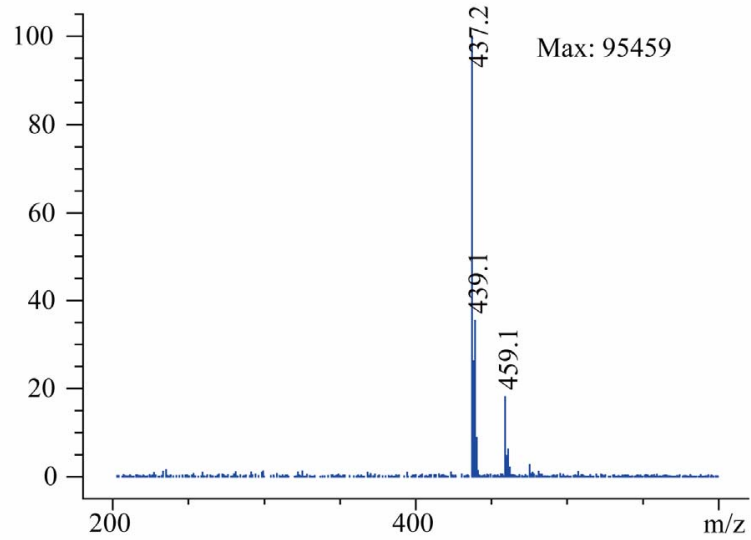

(b)

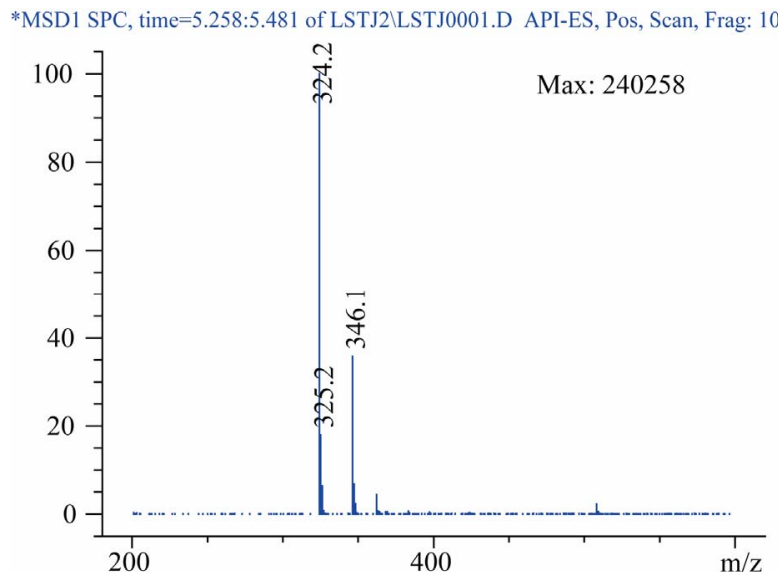

(d)

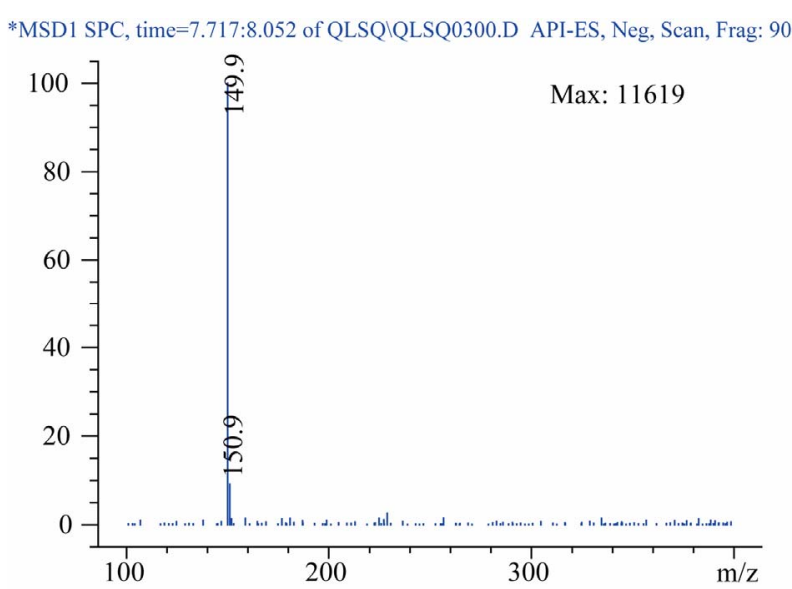

(e)

Figure 2. Product ion spectrum:losartan (a), E3174 (b), hydrochlorothiazide (c), gliclazide (d), paracetamol (e). 

Losartan/Hydrochlorothiazide in Healthy Chinese Male Volunteers
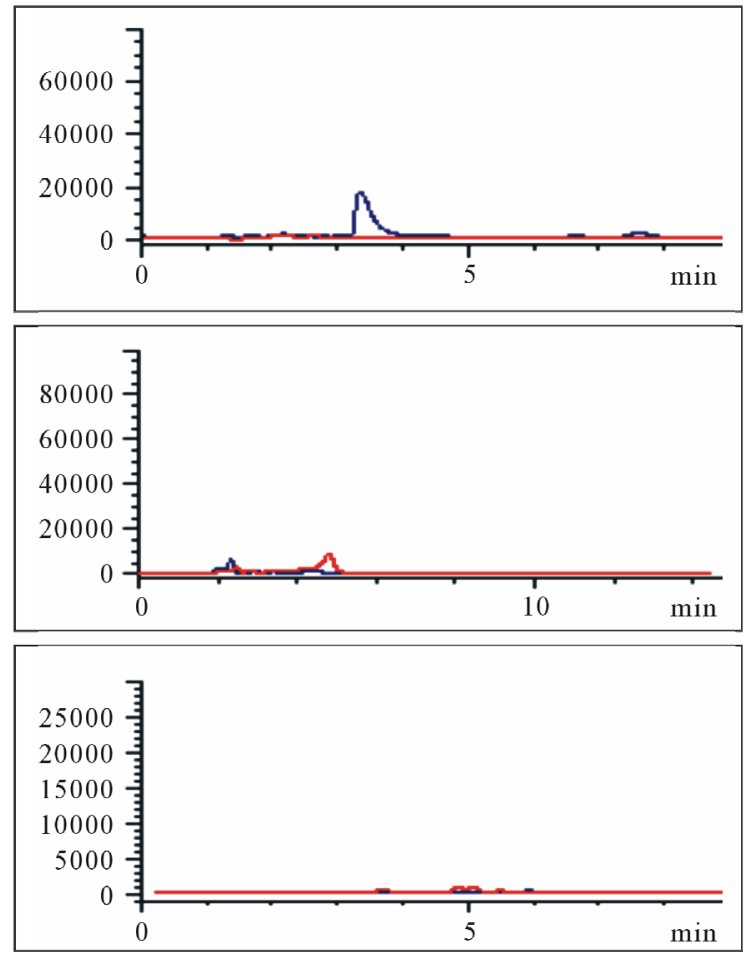

(a)
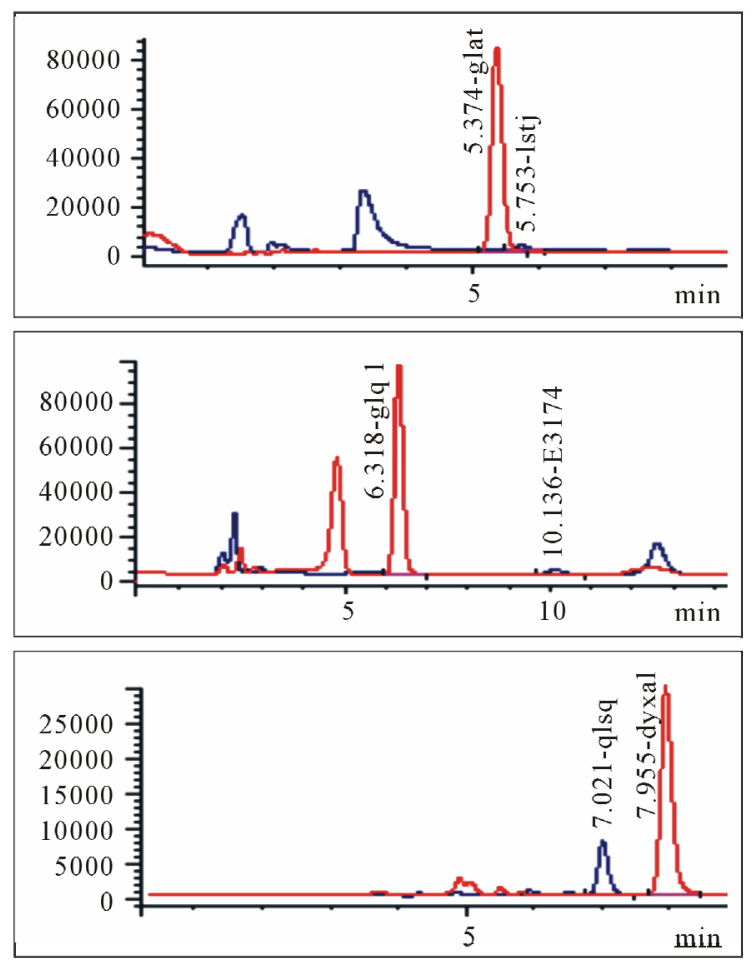

(c)
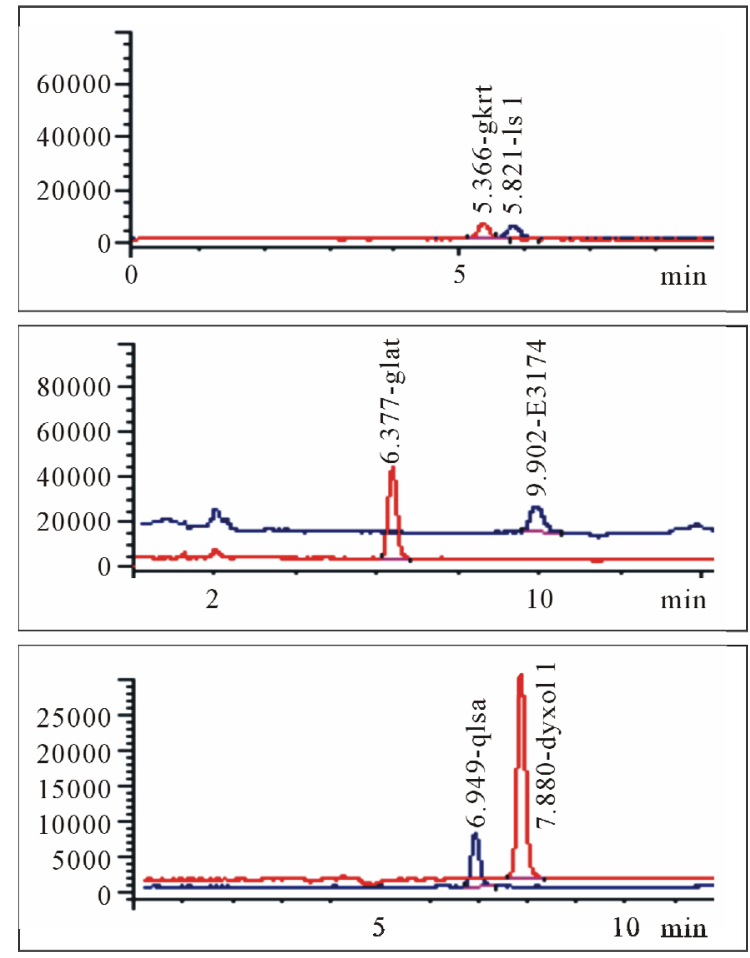

(b)
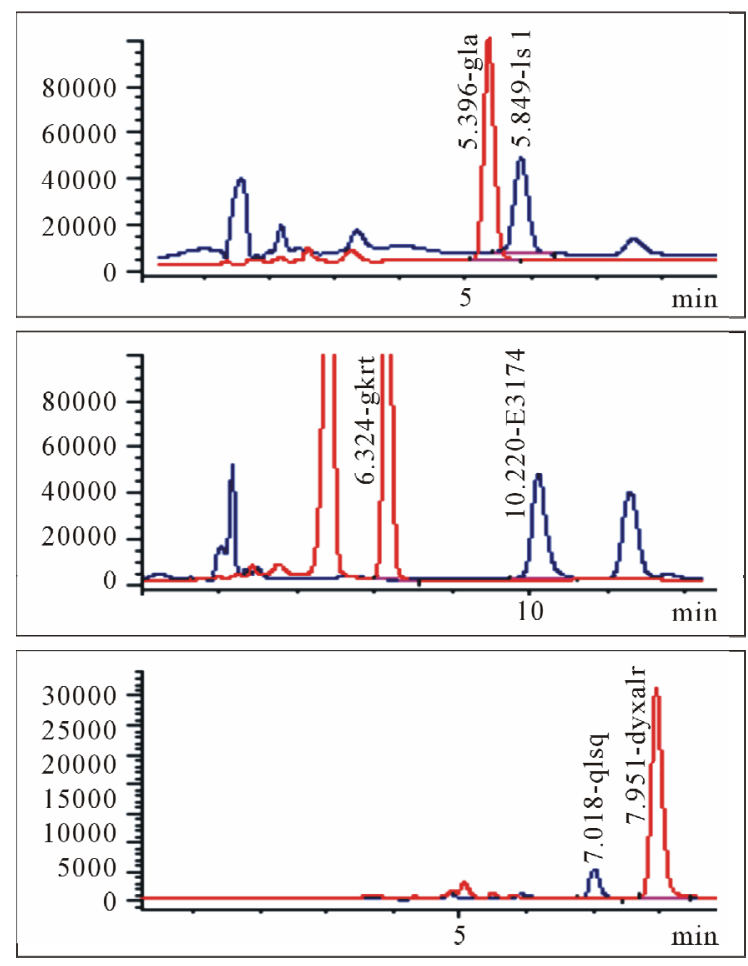

(d)

Figure 3. Blank plasma samples (a), typical chromatograms of losartan and gliclazide, E3174 and gliclazide, hydrochlorothiazide and paracetamol (b), blank plasma spiked with losartan and gliclazide, E3174 and gliclazide, hydrochlorothiazide and paracetamol (c), plasma sample of a volunteer at 4 hours after administration of $50 \mathbf{~ m g ~ l o s a r t a n / 1 2 . 5 ~ m g ~ h y d r o c h l o r o t h i a z i d e ~}$ combination (d). Three graphs in each group (a, b, c or d) represent losartan and gliclazide, E3174 and gliclazide, hydrochlorothiazide and paracetamol from top to bottom. 
The matrix effects were ranged from $96 \%$ to $108 \%$ at three QC concentrations for losartan, E3174 and hydrochlorothiazide, with appropriate extration recovery. The LLOQ of losartan, E3174 and hydrochlorothiazide showed high Signal/Noise ratio. The RSDs were 5.50\%, $3.01 \%$ and $2.23 \%$, respectively. The intra-day and interday precisions were stable by assessing QC samples of losartan, E3174 and hydrochlorothiazide. The RSD\% were ranged from $0.83 \%$ to $5.53 \%$. It was stable after storage at $-20^{\circ} \mathrm{C}$ for days and two freeze-thaw cycles.

\subsection{Bioequivalence of Losartan, E3174 and Hydrochlorothiazide}

The mean plasma concentration-time profiles in human plasma after a single oral administration of $50 \mathrm{mg}$ compound losartan tablet or a single-dose $50 \mathrm{mg}$ losartan/ $12.5 \mathrm{mg}$ hydrochlorothiazide combination tablet were shown in Figure 4 and Figure 5, respectively.

\subsection{PK Parameters}

The mean PK parameters, such as $\mathrm{t}_{1 / 2}, \mathrm{~T}_{\max }, \mathrm{AUC}_{0-\mathrm{t}}$ and $\mathrm{AUC}_{0 \text {-inf }}$, of losartan and E3174 in both monotherapy and combination were shown in Table 1.

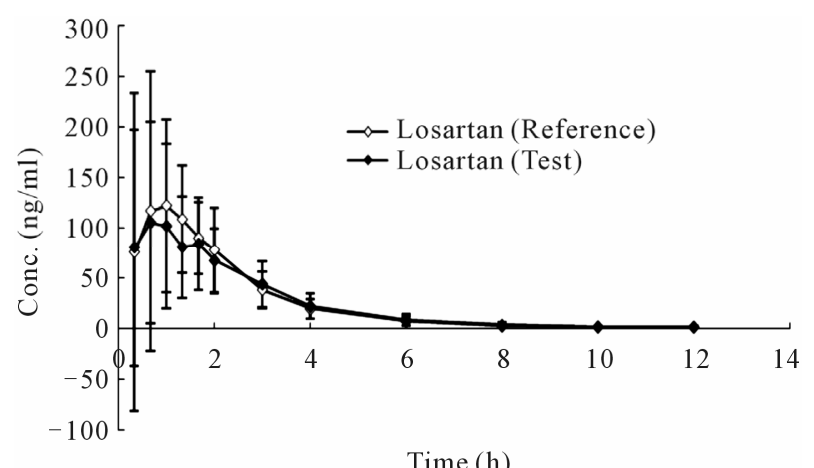

(a)

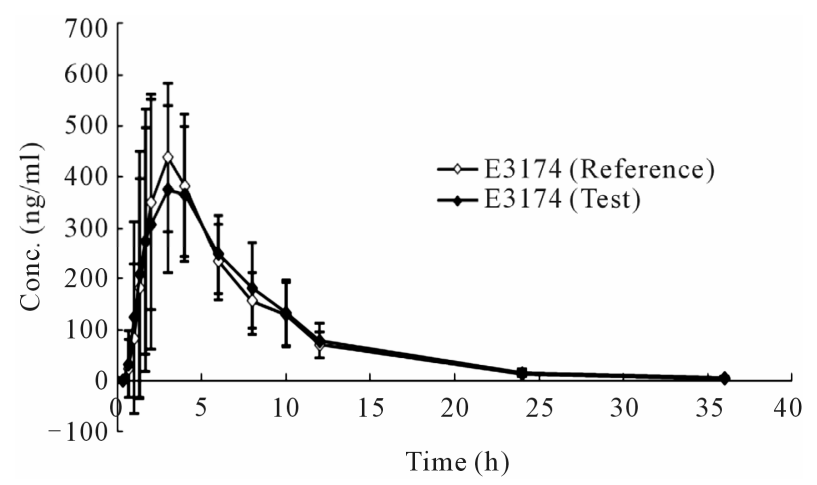

(b)

Figure 4. Concentration-time curves of losartan (a) and E3174 (b) after a single oral administration of monotherapy (Mean \pm SD, $n=20)$.

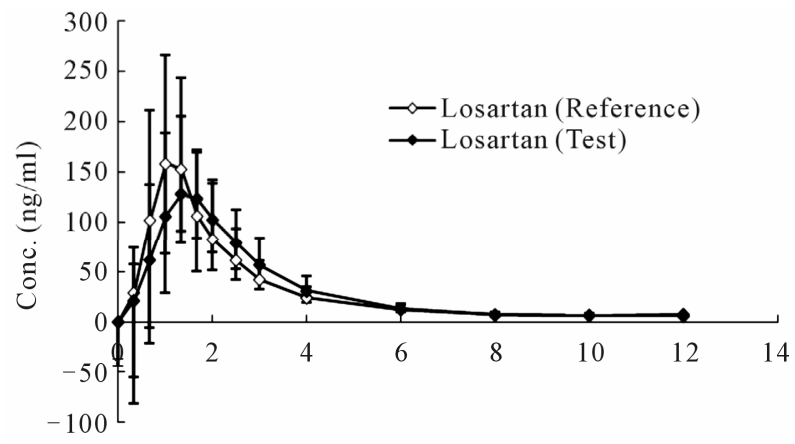

Time $(\mathrm{h})$

(a)

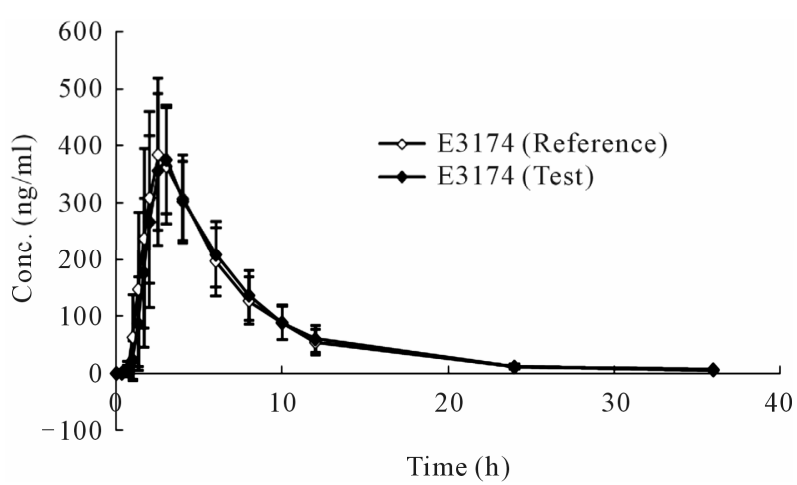

(b)

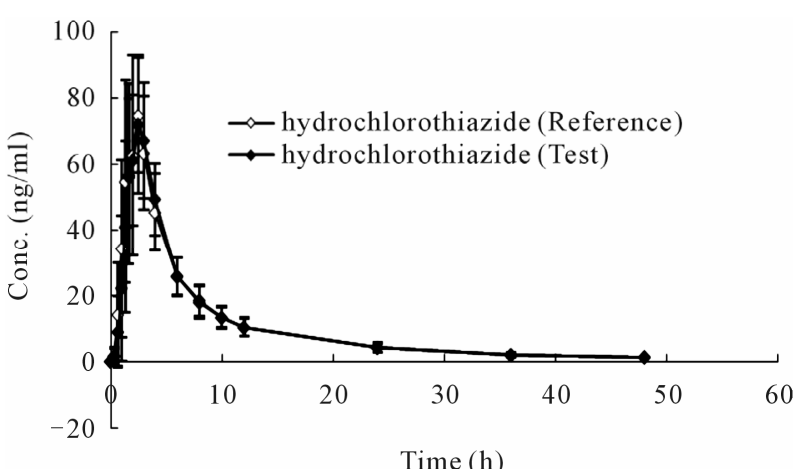

(c)

Figure 5. Concentration-time curves of losartan (a), E3174 (b) and hydrochlorothiazide (c) after a single oral administration of combination (Mean $\pm S D, n=20)$.

\subsection{Metabolism Genotypes Observation}

The AUC ratio of E3174 and losartan after dosing of 50 mg losartan tablets in 20 subjects was calculated and then sequenced in ascending order (Figure 6).

\subsection{Safety and Tolerance Results}

Losartan and hydrochlorothiazide were generally well tolerated without clinically adverse effects according to physical examination and laboratory tests. No significant changes in haematological or biochemical values were 
Table 1. Mean derived pharmacokinetic profiles of losartan and E3174 after administration of the combination tablet compared with monotherapy (Mean $\pm S D, n=20)$.

\begin{tabular}{ccccc}
\hline & \multicolumn{2}{c}{ Losartan } & \multicolumn{2}{c}{ E3174 } \\
\cline { 2 - 5 } & (monotherapy) & (combination) & (monotherapy) & (combination) \\
\hline $\mathrm{t}_{1 / 2 \mathrm{Z}}(\mathrm{h})$ & $1.257 \pm 0.296$ & $1.776 \pm 0.587 *$ & $4.977 \pm 0.872$ & $4.403 \pm 0.863^{*}$ \\
$\mathrm{~T}_{\max }(\mathrm{h})$ & $1.167 \pm 0.489$ & $1.225 \pm 0.496$ & $2.834 \pm 0.616$ & $2.684 \pm 0.574$ \\
$\mathrm{AUC}_{0-\mathrm{t}}\left(\mathrm{ng} \cdot \mathrm{h} \cdot \mathrm{mL}^{-1}\right)$ & $299.629 \pm 97.400$ & $340.442 \pm 129.374$ & $3137.193 \pm 922.053$ & $2573.121 \pm 795.236^{*}$ \\
$\mathrm{AUC}_{0-\text { inf }}\left(\mathrm{ng} \cdot \mathrm{h} \cdot \mathrm{mL}^{-1}\right)$ & $303.490 \pm 98.087$ & $358.573 \pm 128.495$ & $3172.273 \pm 929.721$ & $2620.848 \pm 813.387 *$ \\
$\mathrm{C}_{\max }\left(\mathrm{ng} \cdot \mathrm{mL}^{-1}\right)$ & $213.067 \pm 135.019$ & $223.056 \pm 87.513$ & $478.524 \pm 168.604$ & $418.671 \pm 115.473$ \\
\hline
\end{tabular}

$* P<0.05$.

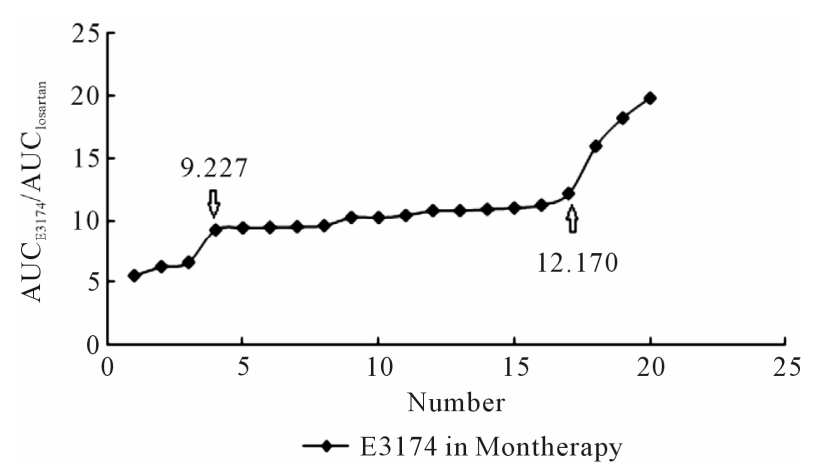

Figure 6. The $\mathrm{AUC}_{\mathrm{E} 3174} / \mathrm{AUC}_{\text {losartan }}$ of E3174 in montherapy.

observed.

\section{Discussion}

\subsection{Bioequivalence Evaluate}

Losartan, with lower side-effects, was approved for hypertension treatment by being treated alone or combined with other agents by FDA in 1995. In the same year, the combination of losartan and low-dose hydrochlorothiazide was approved by FDA, with advantage of better blood pressure-lowering effects for patients whose blood pressure can not be controlled by the monotherapy [2].

After two-one side t-test following ANOVA to $\mathrm{C}_{\max }$, $\mathrm{AUC}_{0-12}$ and $\mathrm{AUC}_{0-\text { inf, no significant difference was }}$ found among losartan, E3174 and hydrochlorothiazide for test and reference formulation of losartan $50 \mathrm{mg}$ and $50 \mathrm{mg}$ losartan/12.5 mg hydrochlorothiazide in 20 healthy volunteers. The $90 \% \mathrm{CI}$ of test and reference mean ratio for $\mathrm{C}_{\max }, \mathrm{AUC}_{0-\mathrm{t}}$ and $\mathrm{AUC}_{0 \text {-inf }}$ were ranged from 80 to $125 \%$. Therefore, it was concluded that the two preparations (test and reference) were bioequivalent.

\subsection{PK Parameters Comparison}

Though it was bioequivalent to combination tablet and monotherapy according to PK parameters of losartan and E3174. The mean $t_{1 / 2}$ of losartan and E3174 was signifi- cantly longer with combination tablet than monotherapy tablet (Table 1, $P<0.05$ ). The mean $\mathrm{AUC}_{0-t}$ and $\mathrm{AUC}_{0 \text {-inf }}$ of E3174 were lower with administration of the combination tablet $(P<0.05)$.

Therefore, firstly it is suggested that hydrochlorothiazide impact the $t_{1 / 2}$ of losartan. Despite this slight delay of losartan, the extent of $\mathrm{C}_{\max }, \mathrm{AUC}_{0-\mathrm{t}}$ and $\mathrm{AUC}_{0 \text {-inf }}$ of losartan were similar when the combination tablet was given, for hydrochlorothiazide may alter the absorption or metabolism of losartan. Secondly, the elimination rate of E3174 was significantly enhanced by using the combination tablet in comparison with monotherapy.

\subsection{Metabolism Genotypes of Losartan}

Losartan is metabolized into active carboxylic acid metabolite (E3174) which has been shown to be more potent at blocking the AT 1 receptor than losartan by CYP2C9. CYP2C9 has several genotypes, two of which are closely relevant to losartan metabolism: CYP2C $9 * 1 / * 1$ (intermediate metabolize) and CYP2C $9 * 1 / * 3$ (extensive metabolize). According to reference, the bioavailability of losartan and E3174 in the CYP2C $9 * 1 / * 1$ subjects and CYP2C $9 * 1 / * 3$ subjects, which are the only two genotypes in Asian, could be due to the interaction of the enzyme activity and losartan plasma concentration profiles [7].

The curve in Figure 6 was apparently divided into three sections at two points (9.227 and 12.170), and they were defined as: the poor, the intermediate and the extensive metabolism. Among 20 volunteers, 3 were poor metabolizers and 3 extensive metabolizers, and the others were intermediate metabolizers. The observed phenotype distribution is similar with previously published studies except in this test there were three genotypes, for there may be a third genotype in Asian. However, the confirmation of CYP genotypes of subjects need further study.

\section{Conclusion}

The losartan tablet and losartan/hydrochlorothiazide 
combination tablet are bioequivalent. The combination of losartan and hydrochlorothiazide significantly alters the pharmacokinetic profiles of losartan tablet with shorter $\mathrm{t}_{1 / 2}$ of $\mathrm{E} 3174$ and lower $\mathrm{AUC}_{0-\mathrm{t}}$ and $\mathrm{AUC}_{0 \text {-inf }}$ of $\mathrm{E} 3174$, paving movement for hypertension treatment. In addition, the observed distribution of phenotype is consistent with previously published studies. The clinical treatment of individual dosage regimen should be implemented, for the pharmacokinetic parameters are quite different in individuals.

\section{REFERENCES}

[1] Z. X. Zhao, Q. X. Wang, E. W. Tsai and X. Z. Qin, "Identification of Losartan Degradates in Stressed Tablets by LC-MS and LC-MS/MS," Journal of Pharmaceutical and Biomedical Analysis, Vol. 20, No. 1-2, 1999, pp. 129-136. doi:10.1016/S0731-7085(99)00004-7

[2] D. J. Triggle, "Angiotensin II Receptor Antagonism: Losartan-Sites and Mechanisms of Action," Clinical Therapeutics, Vol. 17, No. 6, 1995, pp. 1004-1030. doi:10.1016/0149-2918(95)80080-8

[3] T. Tanner, S. Aspley, A. Munn and T. Thomas, "The Pharmacokinetic Profile of a Novel Fixed-Dose Combination Tablet of Ibuprofen and Paracetamol," BMC Clinical Pharmacology, Vol. 10, No. 10, 2010, pp. 989992.

[4] L. H. Liu, P. Z. Liao and J. G. Duan, "Effect of Hyperglycemia on Protein Kinase C in Retina of Diabetic Rats," Journal of Changzhi Medical College, Vol. 3, No. 17, 2003, pp. 3-5.

[5] T. Matsumoto, K. Ishida, K. Kamata, et al., "Mechanisms Underlying the Losartan Treatment-Induced Improvement in the Endothelial Dysfunction Seen in Mesenteric Arteries from Type 2 Diabetic Rats," Pharmacological Research, Vol. 3, 2010, pp. 1-11.

[6] M. Polinko, K. Riffel, H. C. Song and M. W. Lo, "Simultaneous Determination of Losartan and EXP3174 in Human Plasma and Urine Utilizing Liquid Chromatography/ Tandem Mass Spectrometry," Journal of Pharmaceutical and Biomedical Analysis, Vol. 33, No. 1, 2003, pp. 73-84. doi:10.1016/S0731-7085(03)00348-0

[7] R. N. Chena, H. O. Hob, C. Y. Yu and M. T. Sheub, "Development of Swelling/Floating Gastroretentive Drug Delivery System Based on a Combination of $\mathrm{Hy}$ droxyethyl Cellulose and Sodium Carboxymethyl Cellulose for Losartan and Its Clinical Relevance in Healthy Volunteers with CYP2C9 Polymorphism," European Journal of Pharmaceutical Sciences, Vol. 39, No. 1, 2010, pp. 82-89. doi:10.1016/j.ejps.2009.10.015

[8] P. Yang, L. Li, J. Sun and Z. G. He, "Bioequivalent Evaluation of two Losartan/Hydrochlorothiazide Compound Tablets in Healthy Chinese Male Volunteers," Journal of Chinese Pharmaceutical Sciences, Vol. 15, No. 3, 2006, pp. 162-167.

[9] B. Prasaja, L. Sasongko, Y. Harahap, et al., "Simultaneous Quantification of Losartan and Active Metabolite in Human Plasma by Liquid Chromatography-Tandem Mass Spectrometry Using Irbesartan as Internal Standard," Journal of Pharmaceutical and Biomedical Analysis, Vol. 49, 2009, pp. 862-867. doi:10.1016/j.jpba.2009.01.007

[10] P. Yang, Y. J. Wang, L. Li, J. Sun and Z. G. He, "Bioequivalence of Losartan Capsule and Tablet in Healthy Volunteers," The Chinese Journal of Clinical Pharmacology, Vol. 104, 2006, pp. 436-439.

[11] M. Lusina, T. Cindri, J. Tomai, et al., "Stability Study of Losartan/Hydrochlorothiazide Tablets," International Journal of Pharmaceutics, Vol. 291, 2005, pp. 127-137. doi:10.1016/j.ijpharm.2004.07.050

[12] P. Liu, L. Sun, B. J. Wang and R. C. Guo, "Development and Validation of a Sensitive Method for the Determination of Tramadol Plasma and Urine," European Journal of Drug Metabolism and Pharmacokinetics, Vol. 34, 2009, pp. 185-192. doi:10.1007/BF03191172

[13] N. R. Pilli, J. K. Inamadugu and S. Rao, "Simultaneous Determination of Atorvastatin, Amlodipine, Ramipril and Benazepril in Human Plasma by LC-MS/MS and Its Application to a Human Pharmacokinetic Study," Biomedical Chromatography, Vol. 25, No. 4, 2011, pp. 439-449. doi:10.1002/bmc. 1462 